\title{
How Does Social Distancing During COVID-19 Affect Negative Moods and Memory?
}

Weiwei Zhang ${ }^{1}$, Fei Gao ${ }^{2}$, Julien Gross ${ }^{1}$, L. J. Shrum ${ }^{3}$, and Harlene Hayne ${ }^{1}$

${ }^{1}$ Department of Psychology, University of Otago

${ }^{2}$ Department of Marketing, Bentley University

${ }^{3}$ Department of Marketing, HEC Paris

Corresponding Author: Harlene Hayne, Department of Psychology, University of Otago, PO

Box 56, Dunedin, New Zealand 9054. Email: harlene.hayne@otago.ac.nz 


\begin{abstract}
In the absence of an effective vaccine or treatment, the current best defence against COVID19 is social distancing — staying at home as much as possible, keeping distance from others, and avoiding large gatherings. Although social distancing maximizes physical health, we know little about its psychological consequences. In this research $(N=374)$, we investigated the effect of social distancing duration on negative moods and memory. The relation between social distancing duration and both negative mood and memory errors followed the same Ushaped function: negative moods and memory errors initially decreased steadily as social distancing duration increased, at which point ( 30 days) they began to steadily increase.
\end{abstract}

Subsequent analyses indicated that memory errors were mediated by lonely mood in particular. Thus, short-term social distancing might benefit psychological well-being and memory performance, but extended social distancing has the expected negative impact on mood and memory.

Keywords: COVID-19, Social distancing, Social isolation, False memory, Negative mood 
During the first half of 2020, the world has watched as the COVID-19 pandemic has made its way around the globe. At the time of writing this paper, more than 450,000 people have lost their lives (World Health Organization [WHO], n.d.), and the economic damage has been devastating to even the most developed economies. Moreover, there is no clear end in sight, with experts predicting a resurgence of the virus in many areas that have been successful in slowing the spread of the disease so far.

In the absence of an effective vaccine or treatment, the current best defence against COVID-19 is social distancing — staying at home as much as possible, keeping distance from others, and avoiding large gatherings. Although this public health response has proven to be highly effective in containing the spread of the virus and maximizing physical health in countries that have practiced it, it may also come with costs, not only in terms of dollars, but also in terms of psychological well-being. Indeed, psychology has a lot to say about the effects of social isolation (e.g., social exclusion, involuntary incarceration, loneliness), and the negative effects on mental health and well-being are well-documented (e.g., Hawkley \& Cacioppo, 2010; Holt-Lunstad, Smith, Baker, Harris, \& Stephenson, 2015; Morgan, Burns, Fitzpatrick, Pinfold, \& Priebe, 2007). However, the type of social isolation involved in social distancing may be quite different: notwithstanding legal penalties, it is for the most part, voluntary and practiced for self-protection or the protection of vulnerable others; it often involves only "partial isolation" in instances in which family or close friends stay together; and there are now many ways to socialize virtually (social media, videoconferencing, etc.).

Although social distancing in response to a pandemic is not unprecedented, it is nevertheless rare, and consequently psychology does not have a lot to say on the topic. The objective of the current research is to address some of the unanswered questions regarding the psychological impact of atypical social isolation such as social distancing. In particular, we address two issues. One is the effect of social distancing on moods. One intuitive possibility 
is that social distancing leads to increasingly negative moods, like other forms of social isolation such as social exclusion and chronic loneliness. However, as we noted, social distancing in response to a pandemic is not completely involuntary, and not completely socially isolating. Thus, it is plausible that the onset of social distancing may not have a severe negative effect on moods, and could even have possible benefits that result from an escape from a hectic workplace and increased opportunities to spend significant time with family. We explore this question by examining the relation between social distancing duration and negative moods.

The second issue we address is the interrelation between social distancing duration, negative moods, and memory. Research has clearly demonstrated that negative moods can impact memory (for a review, see Kensinger, 2009). For example, experimentally-induced negative moods consistently result in fewer false memories compared to experimentallyinduced neutral and positive moods (e.g., Bookbinder \& Brainerd, 2016; Kensinger \& Schacter, 2006; Storbeck \& Clore, 2005). Little research, however, has addressed how the strength of naturally occurring negative moods influence memory. Here, we examined how changes in negative mood and memory might evolve as the duration of social distancing increases.

A standard procedure for investigating true and false memory performance is the DeeseRoediger-McDermott (DRM) paradigm (Deese, 1959; Roediger \& McDermott, 1995). In this paradigm, people learn word lists in which all the studied words (e.g., bed, rest, pillow) are associated with a critical lure (e.g., sleep) that is semantically related to the words but not presented as part of the list. On a subsequent recognition test, three types of test words are presented: studied words, critical lures, and unrelated words (e.g., boy). Memory for each type of word corresponds to veridical memory, reconstructive-memory errors, and randommemory errors, respectively. 
On the surface, both random-memory errors and reconstructive-memory errors reflect the imperfect nature of human memory, but these two kinds of memory errors are fundamentally different. According to Activation-Monitoring Theory (Roediger, Watson, McDermott, \& Gallo, 2001), the generation of random-memory errors is due to a failure of source monitoring, whereas the generation of reconstructive-memory errors is due to two processes: spreading activation and source monitoring. That is, in the DRM paradigm, semantic activation of studied words spreads to semantically related but unpresented information (the critical lure). As a result, the related information is activated even though it was not part of the original word list. The process of source monitoring helps distinguish between information that has actually been presented and information that has not. But this process is not always successful; when spreading activation occurs and source monitoring fails, false memories occur.

Research has shown that negative moods (compared to neutral and positive moods) tend to impede the kind of relational processing that results in a semantic link between studied words and critical lures in the DRM paradigm. As a result, reconstructive-memory errors are reduced in negative moods (for a review, see Bookbinder \& Brainerd, 2016). Given this, we would predict a negative relation between negative moods induced by social distancing and reconstructive-memory errors. In other memory paradigms, however, negative moods have been shown to supress information monitoring (e.g., Cacioppo, Ernst et al., 2000; Hajcak, McDonald, \& Simons, 2004; Luu, Collins, \& Tucker, 2000). If this effect also occurs in the DRM paradigm, we would predict a positive relation between negative moods and reconstructive-memory errors. Thus, given that semantic activation and monitoring processes are differentially affected by negative moods, it is difficult to make a clear prediction on the impact of negative moods on reconstructive-memory errors. In contrast, given that the generation of random-memory errors is predominantly determined by source-monitoring 
processes, we predict that there would be a positive relation between negative moods and random-memory errors.

In the present study, we used the context of the ongoing global pandemic to explore how social distancing duration influences negative moods and memory. We measured participants' social distancing status and states of negative mood and tested memory performance using the DRM paradigm.

\section{Method}

\section{Participants}

In April 2020, during the COVID-19 outbreak, we recruited participants using the online participant panel, Prolific. We only recruited participants whose Prolific approval rating was greater than $95 \%$. Given that we did not have an ideal reference for determining sample size, we followed Lindsay’s (2015) and Schönbrodt and Perugini’s (2013) recommendations that sample size should approach 250 for stable estimates for interpreting correlations. Our sample consisted of 374 native English speakers (186 men, 188 women; $M_{\text {age }}=29.70$ years, $S D=10$, range $\left.=18-65\right)$ primarily from the U.K. and U.S. The majority of participants lived in a shared-living arrangement (91.18\%) and the remainder lived alone (8.82\%). Each participant received the equivalent of $£ 2.50$ in their local currency for their participation.

\section{Measures and procedure}

Participants answered questionnaires that measured social distancing, negative moods, and memory. The study took approximately $15 \mathrm{~min}$ to complete. Verbatim materials are provided in the Supplemental Material.

The duration of participants' social distancing was measured by asking if they were currently practicing social distancing $(0=$ no, $1=$ yes $)$; if they answered yes, we asked how many days had they been practicing. 
We measured participants' memory performance using the DRM paradigm. During the study phase, participants were shown six, semantically related word lists (see Roediger et al., 2001; Zhang, Gross, \& Hayne, 2017). The word lists were presented in a random order for each participant, one word at a time, with a presentation rate of $2 \mathrm{~s}$ per word. To focus participants' attention between each word, a red cross (“+”) was presented for $800 \mathrm{~ms}$. Participants were asked to remember all of the presented words. To clear their short-term memory, participants then watched a neutral video clip from a National Geographic documentary about lions that was 4 mins, $30 \mathrm{~s}$ in duration. None of the words in the word lists were contained in the video. Finally, participants were shown 36 test words and asked to judge whether each word had been presented during the study phase ("old") or not ("new"). There were three types of test words: studied words that corresponded to veridical memory (three words from each word list, 18 in total), critical lures that corresponded to reconstructive-memory errors ( 1 critical lure was semantically related to each of the six word lists, 6 in total), and unrelated words that corresponded to random-memory errors (words that had not been presented during the study phase, 12 in total).

We measured negative moods by asking participants to rate how lonely, anxious, and depressed they were feeling $(1=$ not at all, $7=$ very $)$. Participants also provided basic demographic information (age, gender, country of residence, and whether they lived with others).

\section{Results}

All data and stimuli are anonymously posted and publicly available at https://osf.io/yfvw7/?view_only=93dc4729f54d4da5b508cf4a6ddf8aa7. We analyzed the data only after we had completed data collection. Given that correlations can be greatly exaggerated by a very few outliers (Lindsay, 2015), we excluded the data from one participant because he reported that he had already been experiencing social distancing for 90 
days; this duration was more than 3 standard deviations above the mean social distancing days for the rest of the sample. Descriptive statistics for the primary variables are presented in Table 1. Figures 1 and 2 depict scatterplots of individuals' social distancing duration and negative mood and memory performance, respectively.

Table 1.

Descriptive Statistics $(N=373)$

\begin{tabular}{lcccc}
\hline Variable & $M$ & $S D$ & Min & Max \\
\hline Social distancing days & 24.94 & 15.43 & 0 & 60 \\
Veridical memory & .77 & .16 & 0 & 1 \\
Reconstructive-memory errors & .73 & .27 & 0 & 1 \\
Random-memory errors & .15 & .19 & 0 & 1 \\
Negative mood composite & 3.96 & 1.51 & 1 & 7 \\
Lonely mood & 3.76 & 1.91 & 1 & 7 \\
Anxious mood & 4.25 & 1.77 & 1 & 7 \\
Depressed mood & 3.87 & 1.72 & 1 & 7 \\
\hline
\end{tabular}

Note: Negative mood composite refers to the composite measure of lonely mood, anxious mood, and depressed mood $(\alpha=.79)$. Veridical memory, reconstructive-memory errors, and random-memory errors refer to the proportion of studied words, critical lures, and unrelated words recognized as "old," respectively.

\section{Negative mood as a function of social distancing}

First, we tested whether social distancing duration increased people's negative mood. A series of four linear regressions with social distancing duration (days) as the independent variable and negative mood (the negative mood composite, lonely mood, anxious mood, or depressed mood) as the dependent variable, respectively, did not support a simple positiveand-linear relation between social distancing duration and negative mood. Detailed results are shown in the Supplementary Material. 
Next, we tested whether the influence of social distancing duration on negative mood was nonlinear. To do this, we conducted a series of quadratic regressions with social distancing duration as the independent variable and the four negative mood variables as the dependent variables. Detailed results are shown in Table 2. The explanatory power of the quadratic models was consistently greater than for the linear models across each of the dependent variables. As shown in Figure 1, the relation between social distancing duration and negative moods followed a U-shaped function — negative moods initially decreased as social distancing duration increased and then at an inflection point occurring between 25 and 35 days, negative moods increased. The same pattern was observed for the negative mood composite and for each of the negative moods separately.

Table 2.

The Relation Between Social Distancing Duration and Negative Mood (Quadratic Model)

\begin{tabular}{|c|c|c|c|c|c|c|c|}
\hline DV & IV & $\beta$ & $S E$ & $t$ & $p$ & $95 \% \mathrm{CI}$ & $R^{2}$ \\
\hline \multirow{2}{*}{$\begin{array}{l}\text { Negative Mood } \\
\text { Composite }\end{array}$} & SD_Days & -.052 & .016 & -3.23 & .001 & {$[-.0834,-.0202]$} & \multirow{2}{*}{.032} \\
\hline & SD_Days $^{2}$ & .001 & .0003 & 2.79 & .006 & {$[.0003, .0015]$} & \\
\hline \multirow{2}{*}{ Lonely Mood } & SD_Days & -.067 & .019 & -3.50 & .001 & {$[-.1047,-.0294]$} & \multirow{2}{*}{.034} \\
\hline & SD_Days $^{2}$ & .001 & .0004 & 3.12 & .002 & {$[.0004, .0019]$} & \\
\hline \multirow{2}{*}{ Anxious Mood } & SD_Days & -.044 & .019 & -2.27 & .024 & {$[-.0816,-.0058]$} & \multirow{2}{*}{.017} \\
\hline & SD_Days $^{2}$ & .001 & .0004 & 2.36 & .019 & {$[.0002, .0016]$} & \\
\hline \multirow{2}{*}{ Depressed Mood } & SD_Days & -.045 & .018 & -2.52 & .012 & {$[-.0796,-.0098]$} & \multirow{2}{*}{.025} \\
\hline & SD_Days ${ }^{2}$ & .001 & .0004 & 1.81 & .071 & {$[-.00006, .0013]$} & \\
\hline
\end{tabular}

Note: SD_Days $=$ social distancing days; $\mathrm{SD}_{-}$Days ${ }^{2}=$ squared social distancing days, $S E=$ robust standard error. 

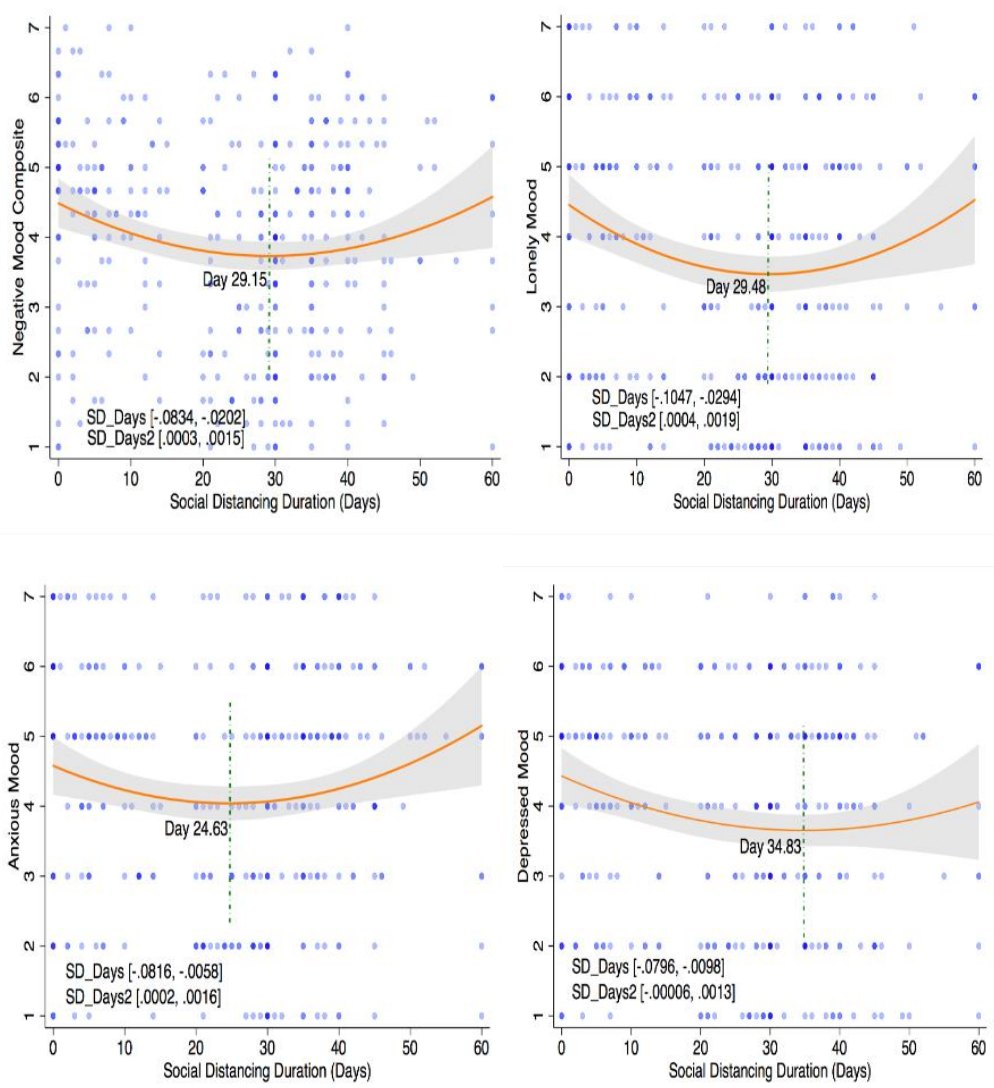

Fig. 1. Scatterplots (with best-fitting regression lines) depicting relations between social distancing duration and negative mood. Confidence bands indicate $95 \%$ confidence intervals (CIs). Values in brackets are also 95\% CIs.

\section{Memory performance as a function of social distancing}

To test the relation between social distancing duration and memory performance, we first calculated the proportion of studied words, critical lures, and unrelated words, recognized as "old" as indexes of veridical memory, reconstructive-memory errors, and random-memory errors, respectively. Then, we conducted both linear and quadratic regressions to explore the relation between social distancing duration and memory performance. Full results for the linear and the quadratic models are shown in Tables 3a and $3 b$, respectively. We will only discuss the results from the model that has a greater explanatory power for each of the dependent variables below. 
Table 3a.

The Relation Between Social Distancing Duration and Memory Performance (Linear Model)

\begin{tabular}{lccccccc}
\hline DV & IV & $\beta$ & $S E$ & $t$ & $p$ & $95 \%$ CI & $R^{2}$ \\
\hline Veridical Memory & SD_Days & .001 & .0005 & 2.51 & .013 & {$[.0003, .0024]$} & .016 \\
$\begin{array}{l}\text { Reconstructive- } \\
\text { Memory Errors }\end{array}$ & SD_Days & -.0002 & .0009 & -.26 & .80 & {$[-.0019, .0015]$} & .0002 \\
$\begin{array}{l}\text { Random-Memory } \\
\text { Errors }\end{array}$ & SD_Days & -.001 & .0008 & -.98 & .33 & {$[-.0024, .0008]$} & .004 \\
\hline
\end{tabular}

Note: SD_Days = social distancing days; $S E=$ robust standard error.

Table 3b.

The Relation Between Social Distancing Duration and Memory Performance (Quadratic Model)

\begin{tabular}{|c|c|c|c|c|c|c|c|}
\hline DV & IV & $\beta$ & $S E$ & $t$ & $p$ & $95 \% \mathrm{CI}$ & $R^{2}$ \\
\hline \multirow{2}{*}{ Veridical Memory } & SD_Days & .002 & .002 & 1.24 & .21 & {$[-.0012, .0051]$} & \multirow{2}{*}{.017} \\
\hline & SD_Days $^{2}$ & -.00001 & .00003 & -.44 & .66 & {$[-.00007, .00005]$} & \\
\hline \multirow{2}{*}{$\begin{array}{l}\text { Reconstructive- } \\
\text { Memory Errors }\end{array}$} & SD_Days & -.005 & .002 & -1.90 & .058 & {$[-.0093, .0002]$} & \multirow{2}{*}{.008} \\
\hline & SD_Days ${ }^{2}$ & .0001 & .00004 & 2.07 & .039 & {$[.00001, .0002]$} & \\
\hline \multirow{2}{*}{$\begin{array}{l}\text { Random-Memory } \\
\text { Errors }\end{array}$} & SD_Days & -.009 & .002 & -3.90 & .0001 & {$[-.0136,-.0045]$} & \multirow{2}{*}{.06} \\
\hline & SD_Days $^{2}$ & .0002 & .00005 & 3.31 & .001 & {$[.00007, .0003]$} & \\
\hline
\end{tabular}

Note: SD_Days $=$ social distancing days; $\mathrm{SD}_{\text {DDays }}{ }^{2}=$ squared social distancing days; $S E=$ robust standard error.

As shown in Figure 2, separate quadratic regressions with social distancing duration as the independent variable and reconstructive-memory errors and random-memory errors as the dependent variables yielded a U-shaped relation between social distancing duration and memory errors. That is, for individuals who were at the initial phase of social distancing (i.e., less than approximately 26 days), their reconstructive- and random-memory errors decreased with social distancing duration. For those who had passed the inflection point (i.e., more than 26 days), however, their reconstructive- and random-memory errors increased with social distancing duration. 
In contrast, linear regression with social distancing duration as the independent variable and veridical memory as the dependent variable showed that participants who had experienced social distancing for a longer duration had higher veridical memory than did those whose duration of social distancing was shorter, showing a simple positive, linear relation between social distancing duration and veridical memory.
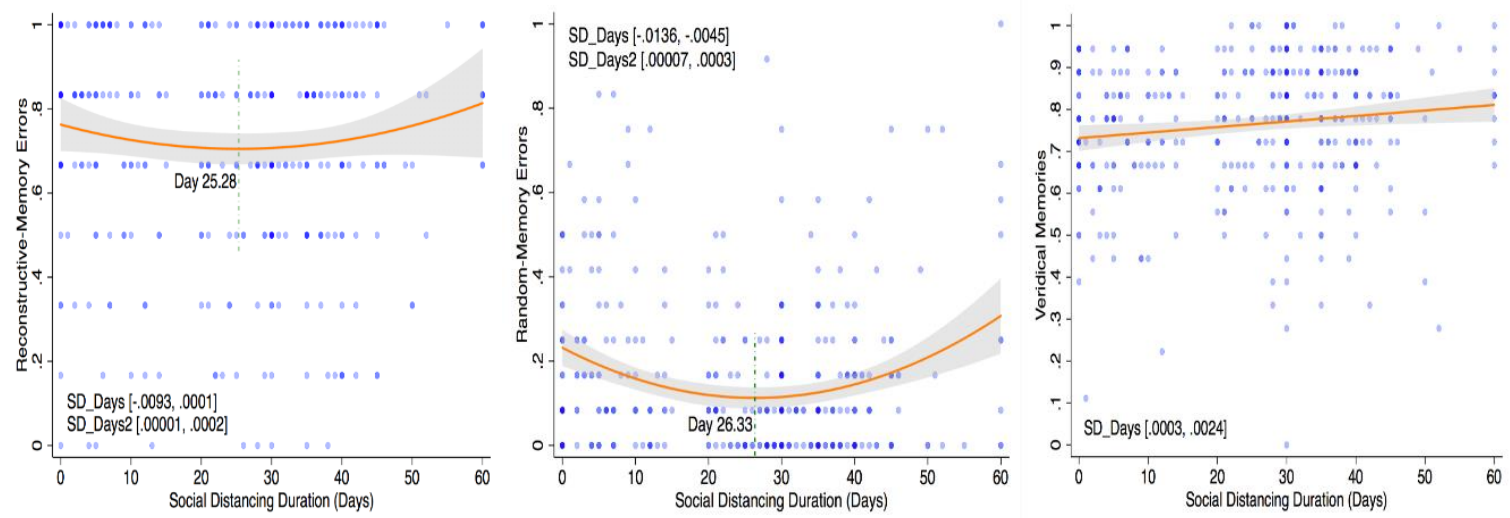

Fig. 2. Scatterplots (with best-fitting regression lines) depicting relationships between social distancing duration and memory performance. Confidence bands indicate $95 \%$ confidence intervals (CIs). Values in brackets are also 95\% CIs.

\section{Lonely mood mediates the effect of social distancing on memory errors}

Finally, we used Hayes' (2018) PROCESS macro to conduct a series of mediation analyses (Model 4, 5,000 bootstrap resamples) to test whether the relation of social distancing duration and memory was mediated by negative moods. The independent variable was either the linear effect of social distancing duration (controlling for the quadratic effect) or the quadratic effect of social distancing duration (controlling for the linear effect). The dependent variable was either veridical memory, reconstructive-memory errors, or random-memory errors. Potential mediators were the four negative mood variables (negative mood composite, lonely mood, anxious mood, and depressed mood).

Only lonely mood mediated the relation between social distancing duration and random-memory errors (see Figure 3a). Similarly, only lonely mood mediated the relation between social distancing duration and reconstructive-memory errors (see Figure 3b, 
marginal significance). None of the other negative moods mediated the relations between social distancing duration and random- and reconstructive-memory errors and veridical memory. Detailed results are shown in the Supplementary Material.

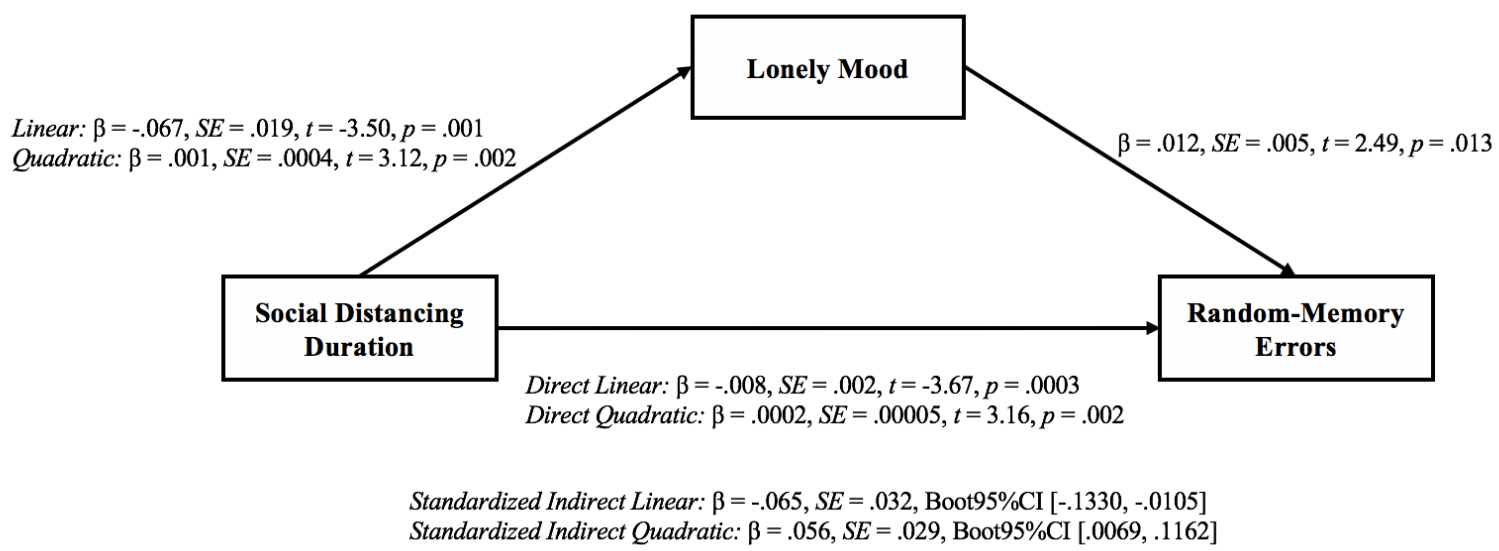

Fig. 3a. Lonely mood mediates the relation between social distancing duration and randommemory errors.

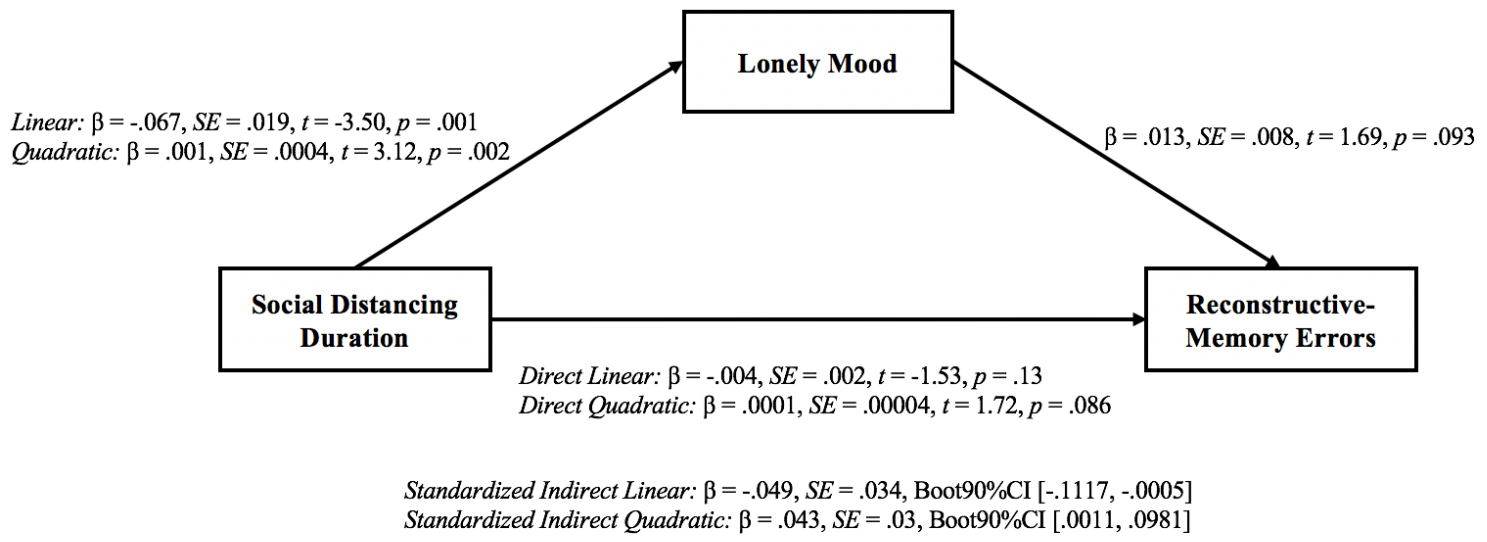

Fig. 3b. Lonely mood mediates the relation between social distancing duration and reconstructive-memory errors. 


\section{Discussion}

Here, we explored the impact of social distancing duration on negative moods and memory. We found that the relation between social distancing duration and negative mood was not a simple one. Negative mood decreased in the initial phase of social distancing. This effect presumably reflected the psychological benefits of a brief escape from a hectic workplace and increased time with family. Considerable research in industrial and organizational psychology has shown that when people have a sense of control or autonomy, their stress is reduced (for reviews, see Sparks, Faragher, \& Cooper, 2001; Ganster, Fusilier, Cooper, \& Robertson, 1989). In this context, working from home as required by social distancing may promote an increased perception of control over the initiation and speed of work. The finding that negative mood declined in the initial phase of social distancing may also reflect increased resilience as a result of spending more time with family-including company, love, and support-which have been shown to predict resilience (for a review, see Masten, Cutuli, Herbers, \& Reed, 2009). Consistent with this idea, more than $90 \%$ of participants were living with their families or others during their social isolation period. In addition, resilience may have also been enhanced by virtual social contacts via technologies, such as Facebook, Skype, and Zoom.

Although social distancing in the short term minimized negative moods, the right-hand side of the U-shaped function illustrates the importance of broader social interactions for long-term mood. According to our findings, after approximately one month of social distancing, negative moods started to increase, approaching the mood states of participants at the initial stages of social distancing. Some moods, such as an anxious mood, even outstripped the original level from Day 50 of practicing social distancing, showing an increasing trend to Day 60. In other words, long-term social distancing may create persistent negative moods which may become an issue of concern. Moreover, compared to short-term 
social distancing, long-term social distancing is more likely to elicit perceived social isolation, which has serious consequences for moods and behavior (e.g., Hawkley \& Cacioppo, 2010; Wilson et al., 2007). Importantly, perceived social isolation is determined by our subjective experience, regardless of objective circumstances (Cacioppo \& Hawkley, 2009). In other words, even though people are not literally alone during the period of social distancing, their perceived social isolation may become stronger with extended social distancing, contributing to a rise in negative mood.

Consistent with research using the same DRM word lists, we observed a strong false memory effect; participants falsely recognized the critical lures at a rate that was indistinguishable from their recognition of words that had been on the lists. To the best of our knowledge, the present study is the first to explore how false memories naturally change with the strength of negative moods. According to the Activation-Monitoring Theory of DRM false memories (Roediger et al., 2001), both semantic activation and source monitoring are likely to determine the generation of reconstructive-memory errors, whereas source monitoring alone determines the generation of random-memory errors. On the one hand, research has shown that negative moods may impede relational processing (e.g., Storbeck \& Clore, 2005, 2011), which in turn impairs semantic activation, reducing reconstructivememory errors. Accordingly, the stronger the negative moods, the fewer reconstructivememory errors. On the other hand, negative moods also supress the monitoring process (e.g., Hajcak et al., 2004; Luu et al., 2000), which in turn increases memory errors regardless of error type. Thus, the stronger the negative moods, the higher the number of reconstructiveand random-memory errors. The findings that reconstructive- and random-memory errors were both positively related to the strength of naturally-changing negative moods, particularly feelings of loneliness, indicate that the effect of a negative mood on memory 
reported here was primarily due to the impact on source monitoring rather than on the impact on semantic activation.

In the present study, why would negative mood effect false memory differently than in prior laboratory-based research (Bookbinder \& Brainerd, 2016)? One possibility might be that in most laboratory-based studies of mood induction, the negative mood has been sadness, not loneliness. In contrast, here it was lonely mood in particular that mediated the association between social distancing duration and reconstructive- and random-memory errors. We know that lonely mood leads to diminished executive control (e.g., Cacioppo, Ernst, et al., 2000; Layden et al., 2017). Given that source monitoring in memory retrieval is a form of executive control (Shimamura, 2002), participants' lonely mood may have impeded their source monitoring, which in turn led to memory errors.

The findings of the current study have important practical implications for current social distancing policies during pandemics such as COVID-19. Our findings suggest that shortterm social distancing $(<\sim 4$ weeks) might benefit people's psychological well-being (by relieving their negative moods). With extended social distancing, however, the tables begin to turn and any of the psychological and cognitive advantages of a brief break from the world, begin to recede. It is important to note that the maximum duration of social distancing in the present study was 60 days. As the pandemic continues, it will be important to chart the impacts of even longer social distancing periods as well as the impacts of a requirement to return to social distancing after a brief period of re-entry into the world. Sadly, until a vaccine is developed, the ongoing presence of COVID-19 will provide ample opportunity to explore these important psychological questions. 


\section{References}

Bookbinder, S., \& Brainerd, C. (2016). Emotion and false memory: The context-content paradox. Psychological Bulletin, 142(12), 1315-1351.

https://doi.org/10.1037/bul0000077

Cacioppo, J. T., Ernst, J. M., Burleson, M. H., McClintock, M. K., Malarkey, W. B., Hawkley, L. C., .. . Hugdahl, K. (2000). Lonely traits and concomitant physiological processes: The MacArthur social neuroscience studies. International Journal of Psychophysiology, 35(2-3), 143-154. https://doi.org/10.1016/S0167-8760(99)00049-5

Cacioppo, J. T., \& Hawkley, L. C. (2009). Perceived social isolation and cognition. Trends in Cognitive Sciences, 13(10), 447-454. https://doi.org/10.1016/j.tics.2009.06.005

Deese, J. (1959). On the prediction of occurrence of particular verbal intrusions in immediate recall. Journal of Experimental Psychology, 58(1), 17-22. https://doi.org/10.1037/h0046671

Ganster, D. C., \& Fusilier, M. R. (1989). Control in the workplace. In C. L. Cooper \& I. T. Robertson (Eds.), International review of industrial and organizational psychology (pp. 235-280). Chichester, UK: Wiley.

Hajcak, G., McDonald, N., \& Simons, R. F. (2004). Error-related psychophysiology and negative affect. Brain and Cognition, 56(2), 189-197. https://doi.org/10.1016/j.bandc.2003.11.001

Hawkley, L. C., \& Cacioppo, J. T. (2010). Loneliness matters: A theoretical and empirical review of consequences and mechanisms. Annals of Behavioral Medicine, 40(2), 218227. https://doi.org/10.1007/s12160-010-9210-8

Hayes, A. F. (2018). Introduction to mediation, moderation, and conditional process analysis: A regression-based approach. New York, NY: Guilford Press. 
Holt-Lunstad, J., Smith, T. B., Baker, M., Harris, T., \& Stephenson, D. (2015). Loneliness and social isolation as risk factors for mortality: A meta-analytic review. Psychological Science, 10(2). 227-237. https://doi.org/10.1177/1745691614568352

Howe, M. L., \& Malone, C. (2011). Mood-congruent true and false memory: Effects of depression. Memory, 19(2), 192-201. https://doi.org/10.1080/09658211.2010.544073

Joormann, J., Teachman, B. A., \& Gotlib, I. H. (2009). Sadder and less accurate? False memory for negative material in depression. Journal of Abnormal Psychology, 118(2), 412-417. https://doi.org/10.1037/a0015621

Kensinger, E. A. (2009). Remembering the details: Effects of emotion. Emotion Review, 1(2), 99-113. https://doi.org/10.1177/1754073908100432

Kensinger, E. A., \& Schacter, D. L. (2006). When the Red Sox shocked the Yankees: Comparing negative and positive memories. Psychonomic Bulletin \& Review, 13(5), 757-763. https://doi.org/10.3758/BF03193993

Layden, E. A., Cacioppo, J. T., Cacioppo, S., Cappa, S. F., Dodich, A., Falini, A., \& Canessa, N. (2017). Perceived social isolation is associated with altered functional connectivity in neural networks associated with tonic alertness and executive control. Neuroimage, 145, 58-73. https://doi.org/10.1016/j.neuroimage.2016.09.050

Lindsay, D. S. (2015). Replication in psychological science. Psychological Science, 26(12), 1827-1832. https://doi.org/10.1177/0956797615616374

Luu, P., Collins, P., \& Tucker, D. M. (2000). Mood, personality, and self-monitoring: negative affect and emotionality in relation to frontal lobe mechanisms of error monitoring. Journal of Experimental Psychology: General, 129(1), 43-60. https://doi.org/10.1037/0096-3445.129.1.43

Masten, A. S., Cutuli, J. J., Herbers, J. E., \& Reed, M.-G. J. (2009). Resilience in development. In S. J. Lopez \& C. R. Snyder (Eds), Oxford library of psychology. 
Oxford handbook of positive psychology (pp. 117-131). New York, NY: Oxford University Press.

Morgan, C., Burns, T., Fitzpatrick, R., Pinfold, V., \& Priebe, S. (2007). Social exclusion and mental health: conceptual and methodological review. The British Journal of Psychiatry, 191(6), 477-483. https://doi.org/10.1192/bjp.bp.106.034942

Roediger, H. L., \& McDermott, K. B. (1995). Creating false memories: Remembering words not presented in lists. Journal of Experimental Psychology: Learning, Memory, and Cognition, 21(4), 803-814. https://doi.org/10.1037/0278-7393.21.4.803

Roediger, H. L., Watson, J. M., McDermott, K. B., \& Gallo, D. A. (2001). Factors that determine false recall: A multiple regression analysis. Psychonomic Bulletin \& Review, 8(3), 385-407. https://doi.org/10.3758/BF03196177

Russo, R., Whittuck, D., Roberson, D., Dutton, K., Georgiou, G., \& Fox, E. (2006). Moodcongruent free recall bias in anxious individuals is not a consequence of response bias. Memory, 14(4), 393-399. https://doi.org/10.1080/09658210500343166

Schönbrodt, F. D., \& Perugini, M. (2013). At what sample size do correlations stabilize? Journal of Research in Personality, 47(5), 609-612. https://doi.org/10.1016/j.jrp.2013.05.009

Shimamura, A. P. (2002). Memory retrieval and executive control. In D. T. Stuss \& R. T. Knight (Eds.), Principles of frontal lobe function (pp. 210-220). New York, NY: Oxford University Press.

Sparks, K., Faragher, B., \& Cooper, C. L. (2001). Well-being and occupational health in the 21st century workplace. Journal of Occupational and Organizational Psychology, 74(4), 489-509. https://doi.org/10.1348/096317901167497 
Storbeck, J., \& Clore, G. L. (2005). With sadness comes accuracy; with happiness, false memory: Mood and the false memory effect. Psychological Science, 16(10), 785-791. https://doi.org/10.1111/j.1467-9280.2005.01615.x

Storbeck, J., \& Clore, G. L. (2011). Affect influences false memories at encoding: Evidence from recognition data. Emotion, 11(4), 981-989. https://doi.org/10.1037/a0022754

World Health Organization. (n.d.). WHO Coronavirus Disease (COVID-19) Dashboard. Retrieved June 18, 2019, from https://covid19.who.int/

Wilson, R. S., Krueger, K. R., Arnold, S. E., Schneider, J. A., Kelly, J. F., Barnes, L. L., . . . Bennett, D. A. (2007). Loneliness and risk of Alzheimer disease. Archives of General Psychiatry, 64(2), 234-240. doi:10.1001/archpsyc.64.2.234

Zhang, W., Gross, J., \& Hayne, H. (2017). The effect of mood on false memory for emotional DRM word lists. Cognition and Emotion, 31(3), 526-537.

doi:10.1080/02699931.2016.1138930 